\title{
Epoxidation of Chiral Camphor $N$-Enoylpyrazolidinones with Methyl(trifluoromethyl)dioxirane and Urea Hydrogen Peroxide/Acid Anhydride: Reversal of Stereoselectivity
}

Chai Ling Fan, Wei-Der Lee, Nai-Wei Teng, Ying-Chieh Sun* and Kwunmin Chen*

\author{
Department of Chemistry, National Taiwan Normal \\ University, Taipei, Taiwan 116, ROC
}
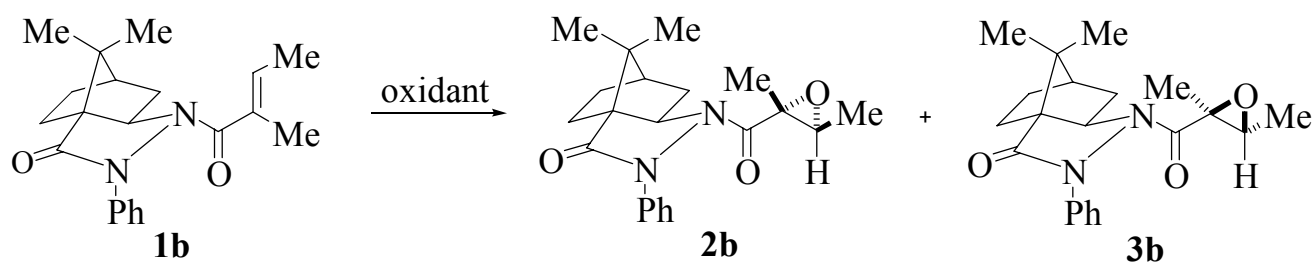

UHP/TFSA

Dioxirane

95

5

$15 \quad: \quad 85$

\section{Supporting Information}

$\left[\left(\right.\right.$ Copies of ${ }^{1} \mathrm{H}$ (2a-d and 3a-d) and ${ }^{13} \mathrm{C}$ (2a-d and 3a-b, 3d) NMR spectra and X-ray data (2a-d and 3b)] 

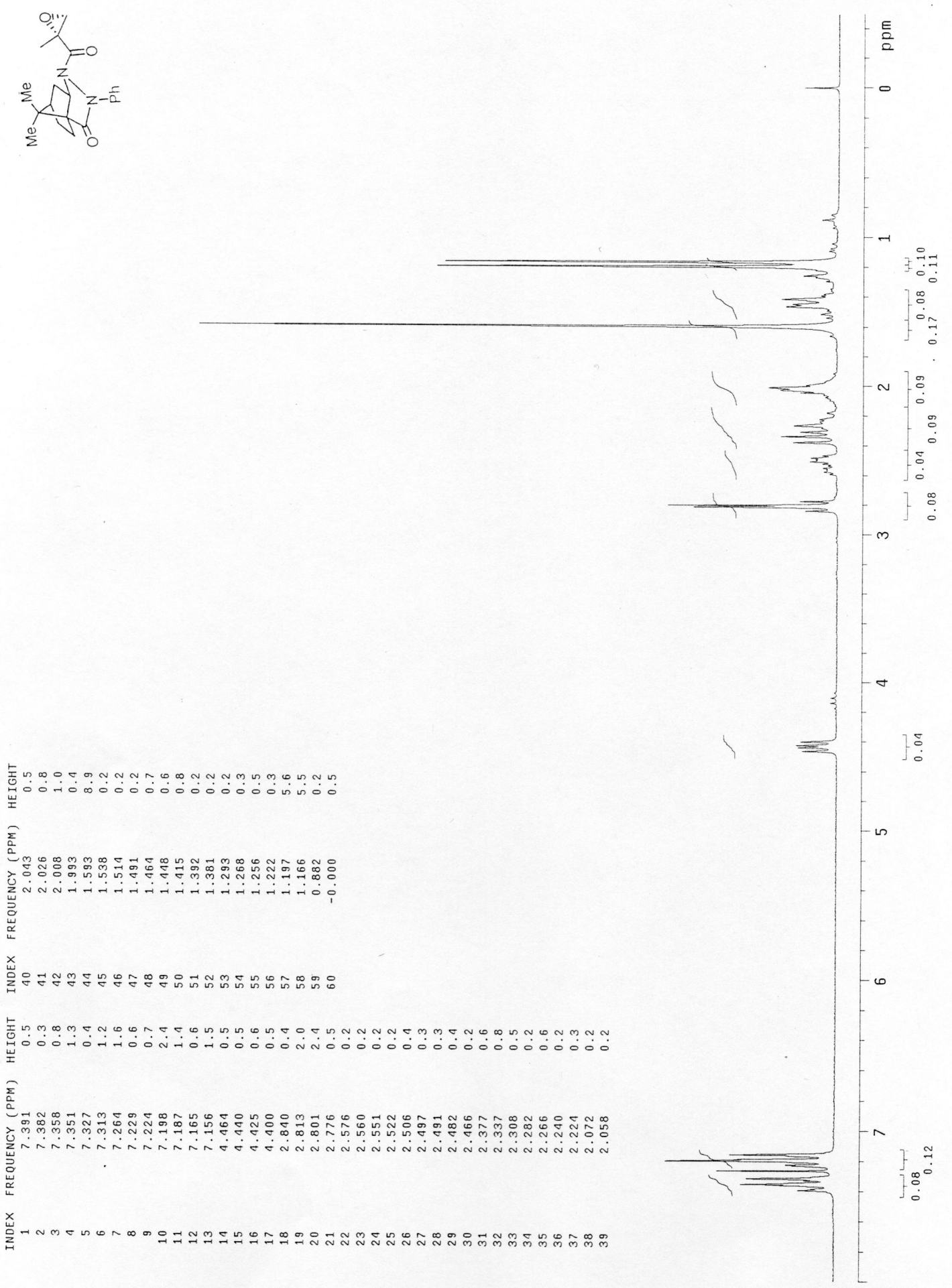


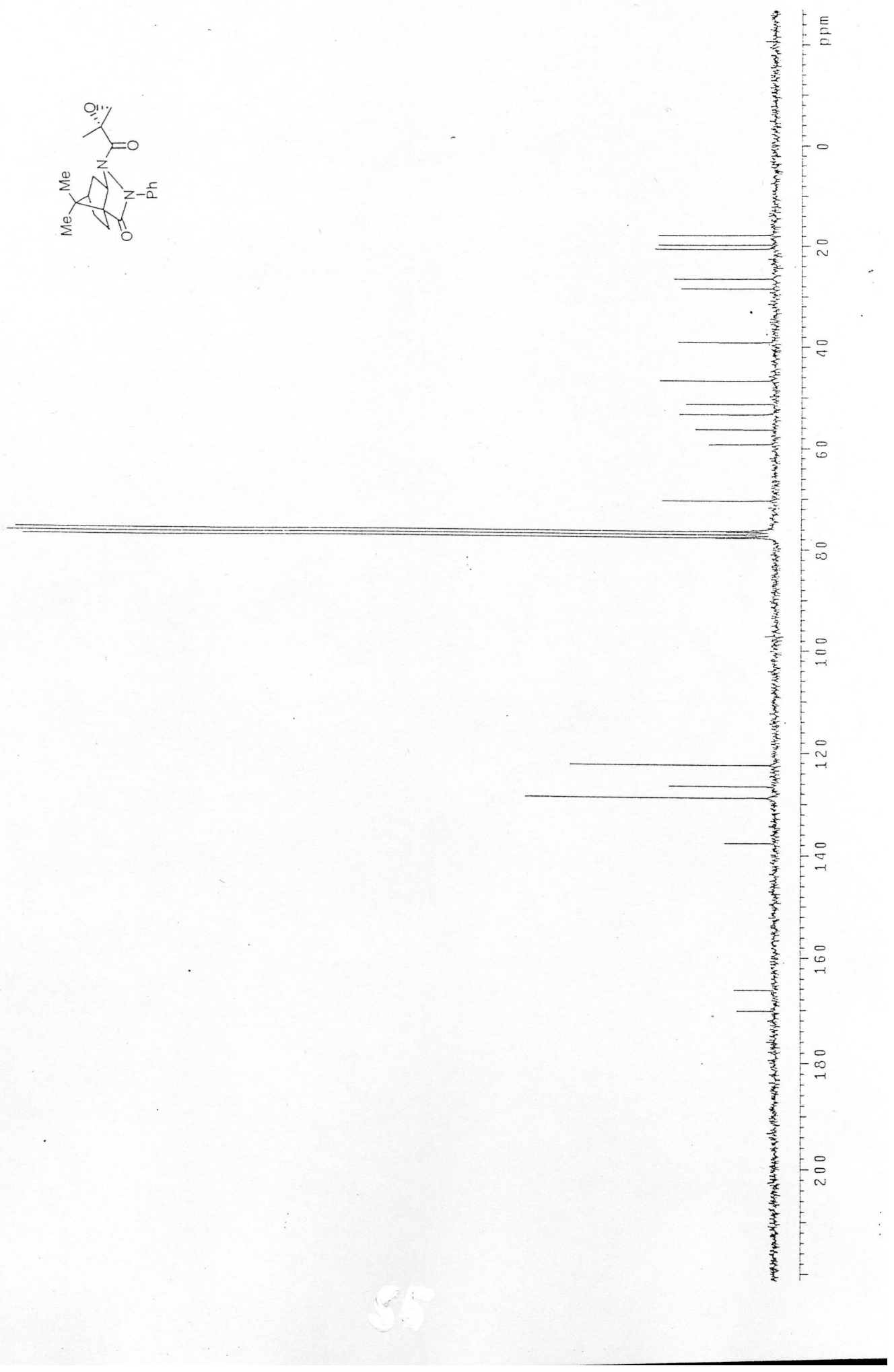



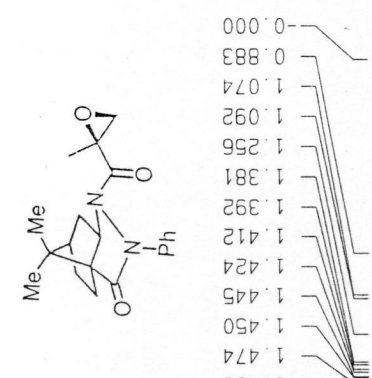

I8०. 1 -

$98 g^{\circ} i$

唱

५96. I -

$\nabla 96^{\circ} \mathrm{I}$

620.5 F

$\nabla 81.5-1$

902 ᄃ

6记元

ธદટ.

$185.5]$

घ6ट.

$\nabla S L ' z-1$

994 'ट

I6 $L$ ' ट -

$508.5]$

$820^{\circ} \varepsilon$

计 $\varepsilon$

$250 \circ$

$\triangle 0 I \cdot \square-$

计政
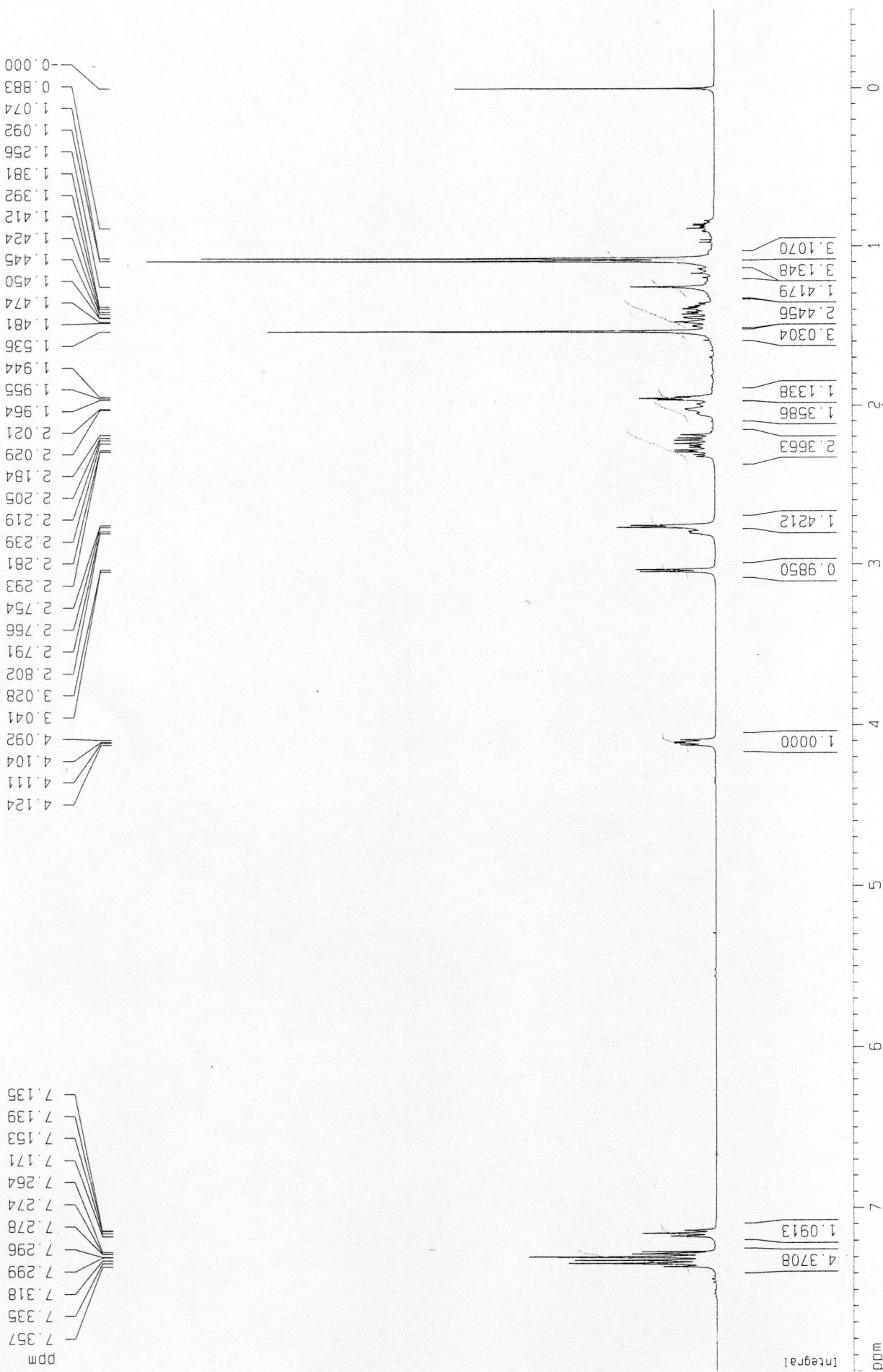

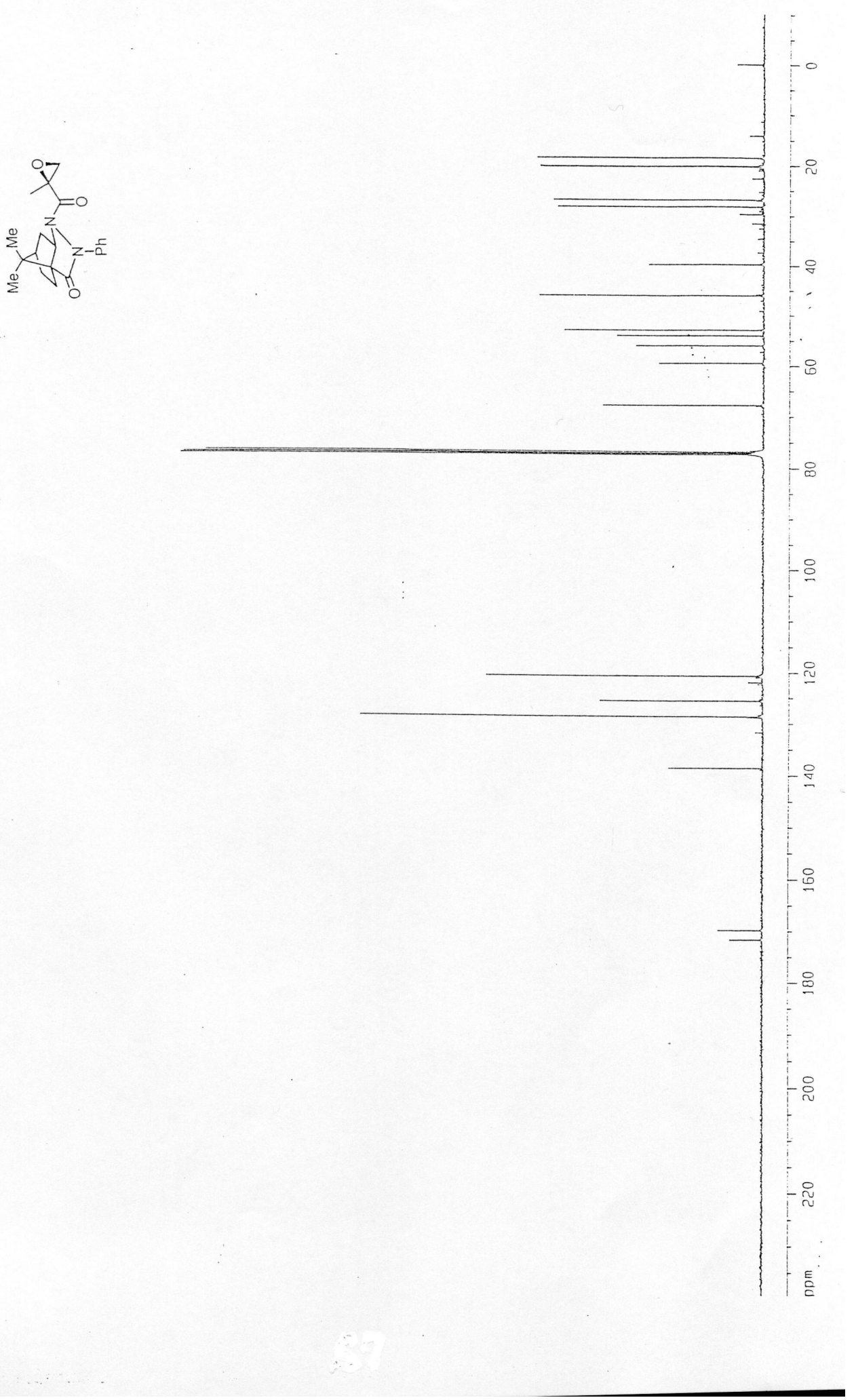

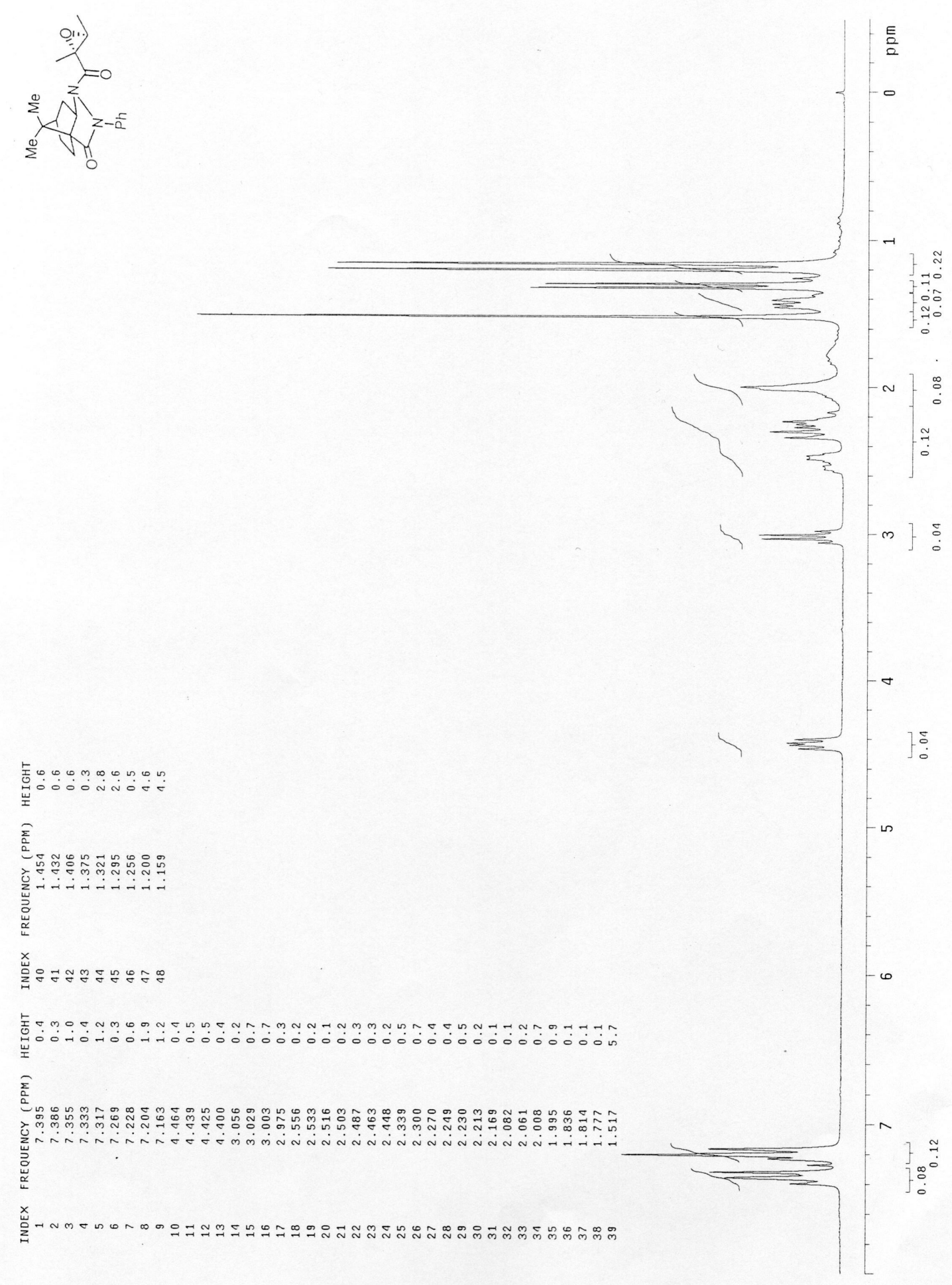

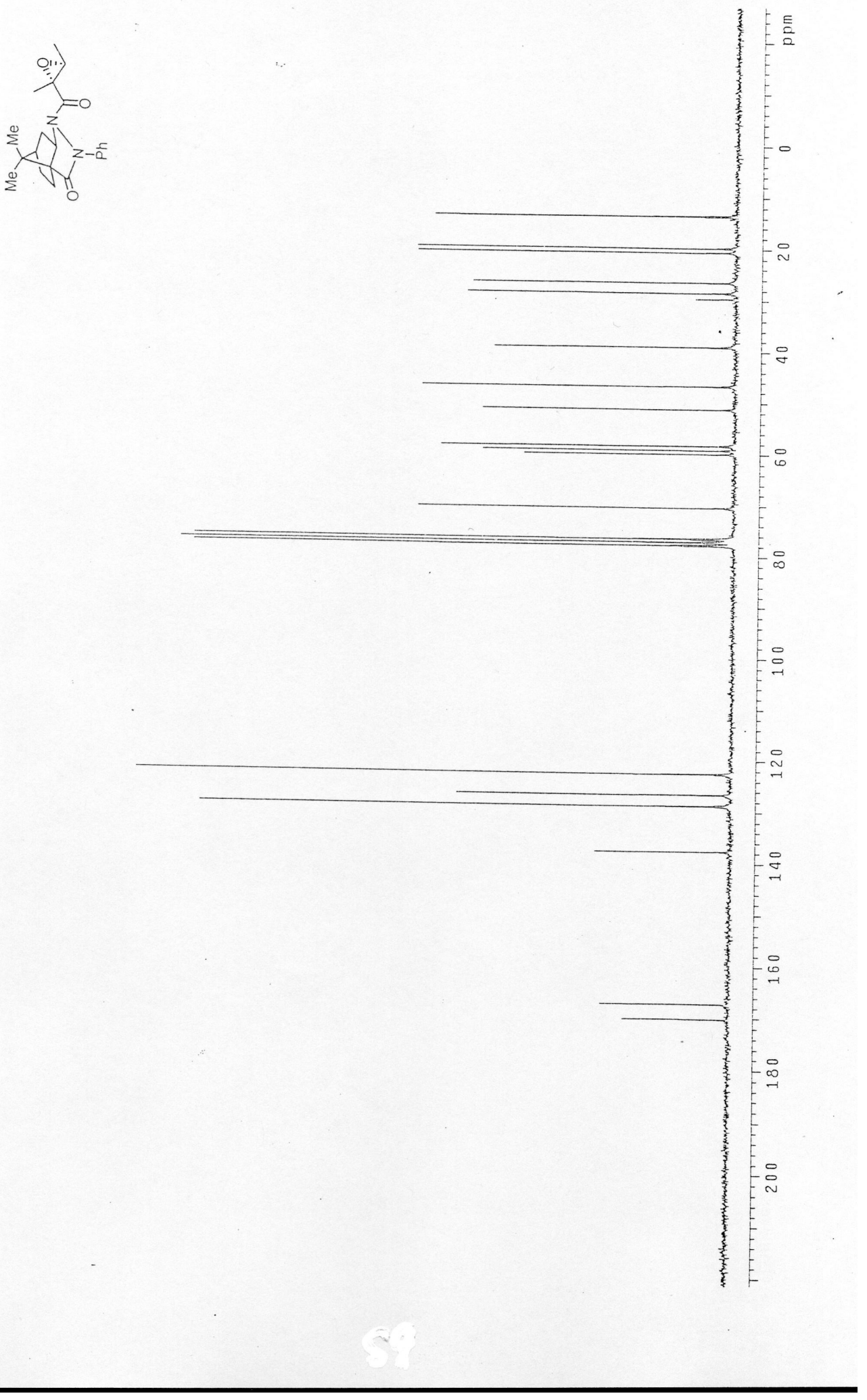

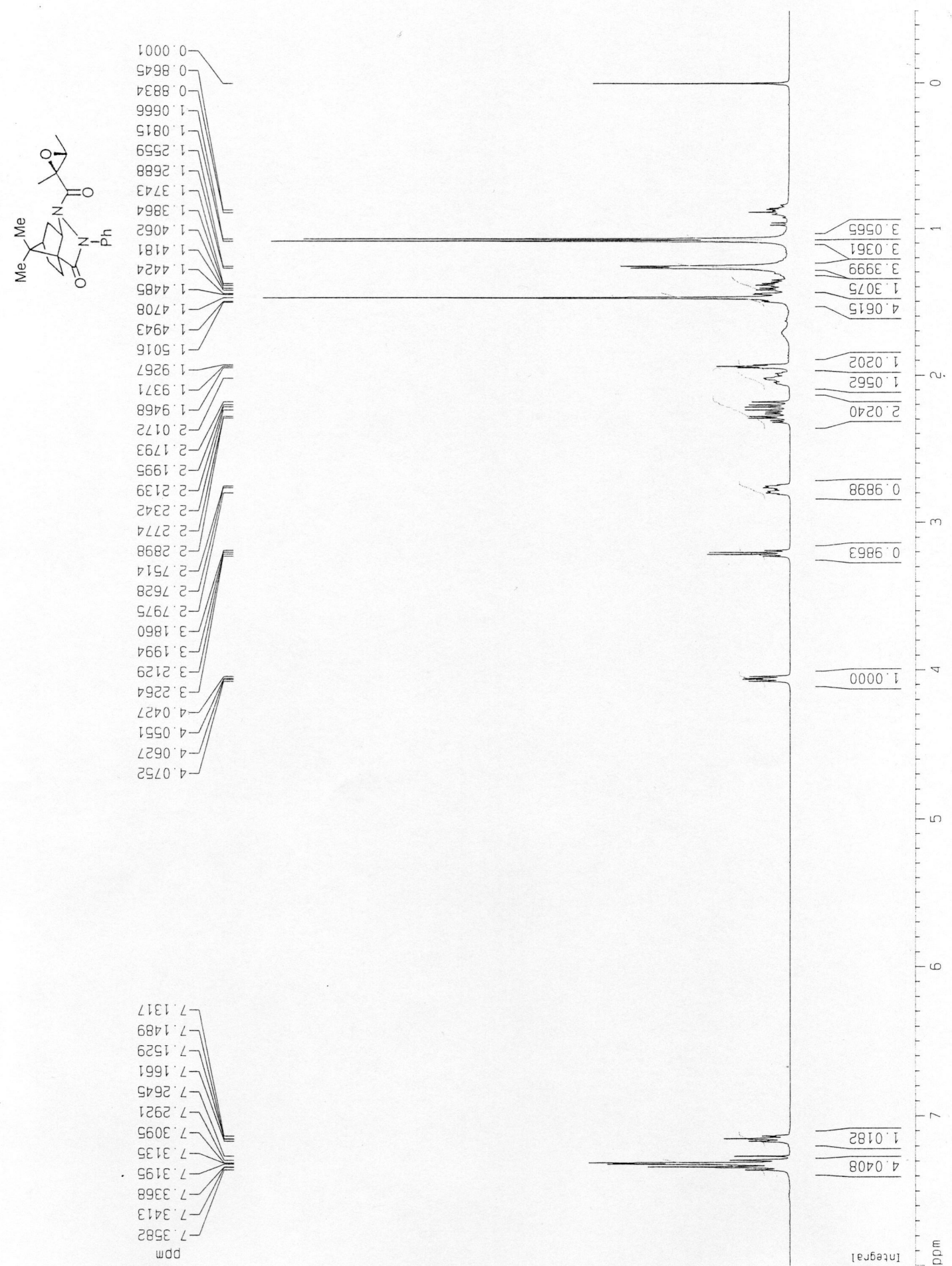

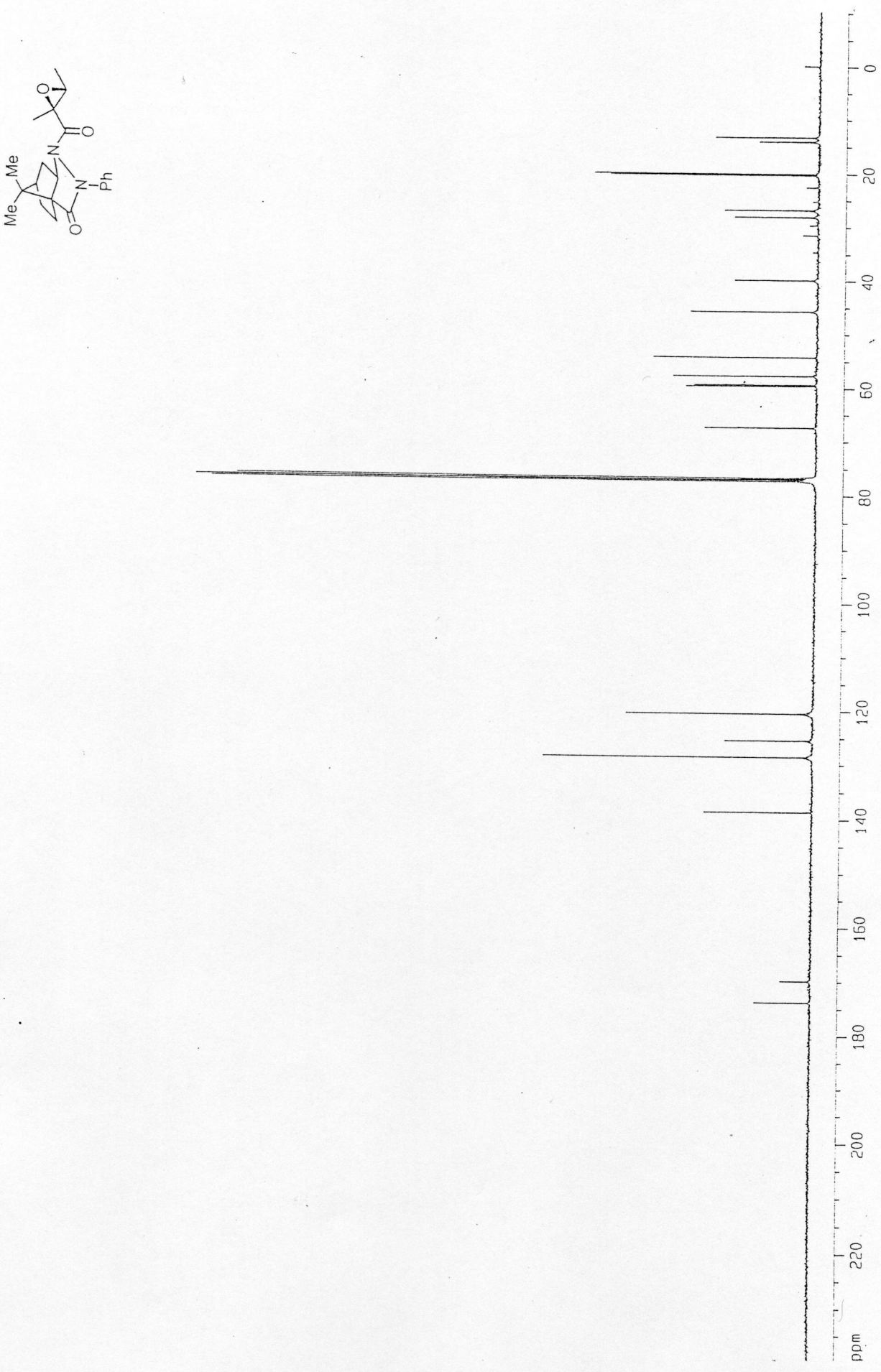


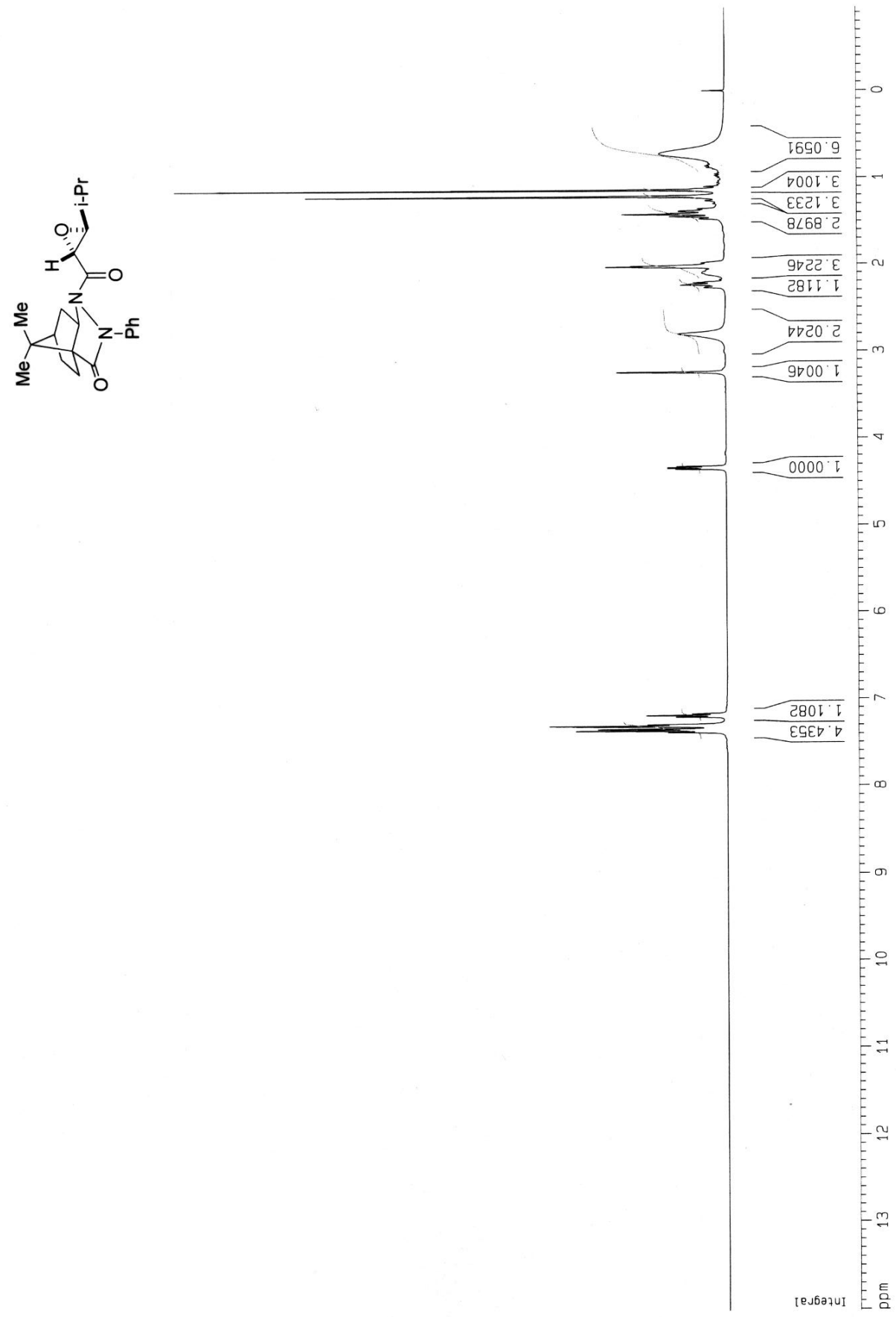




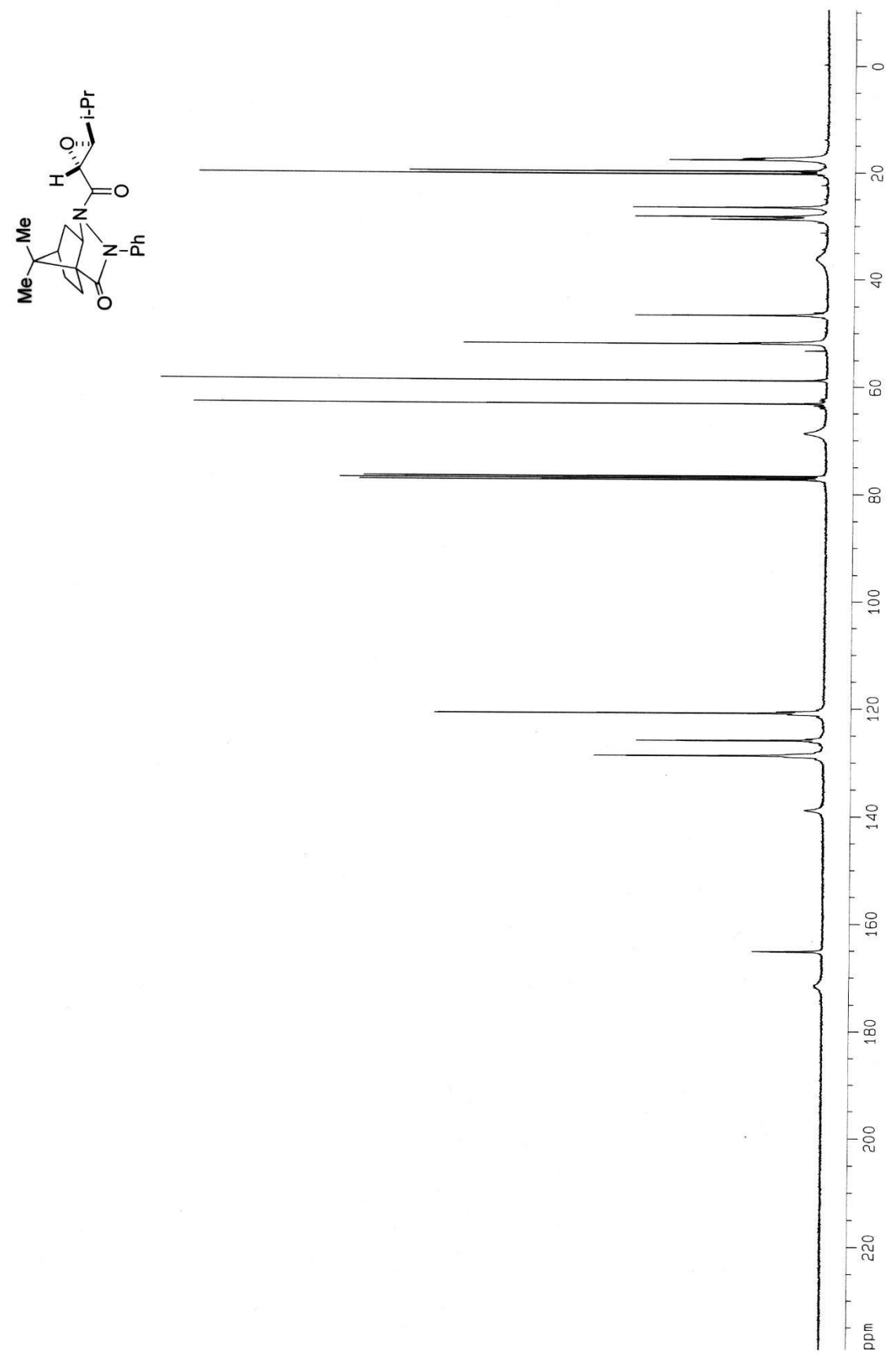




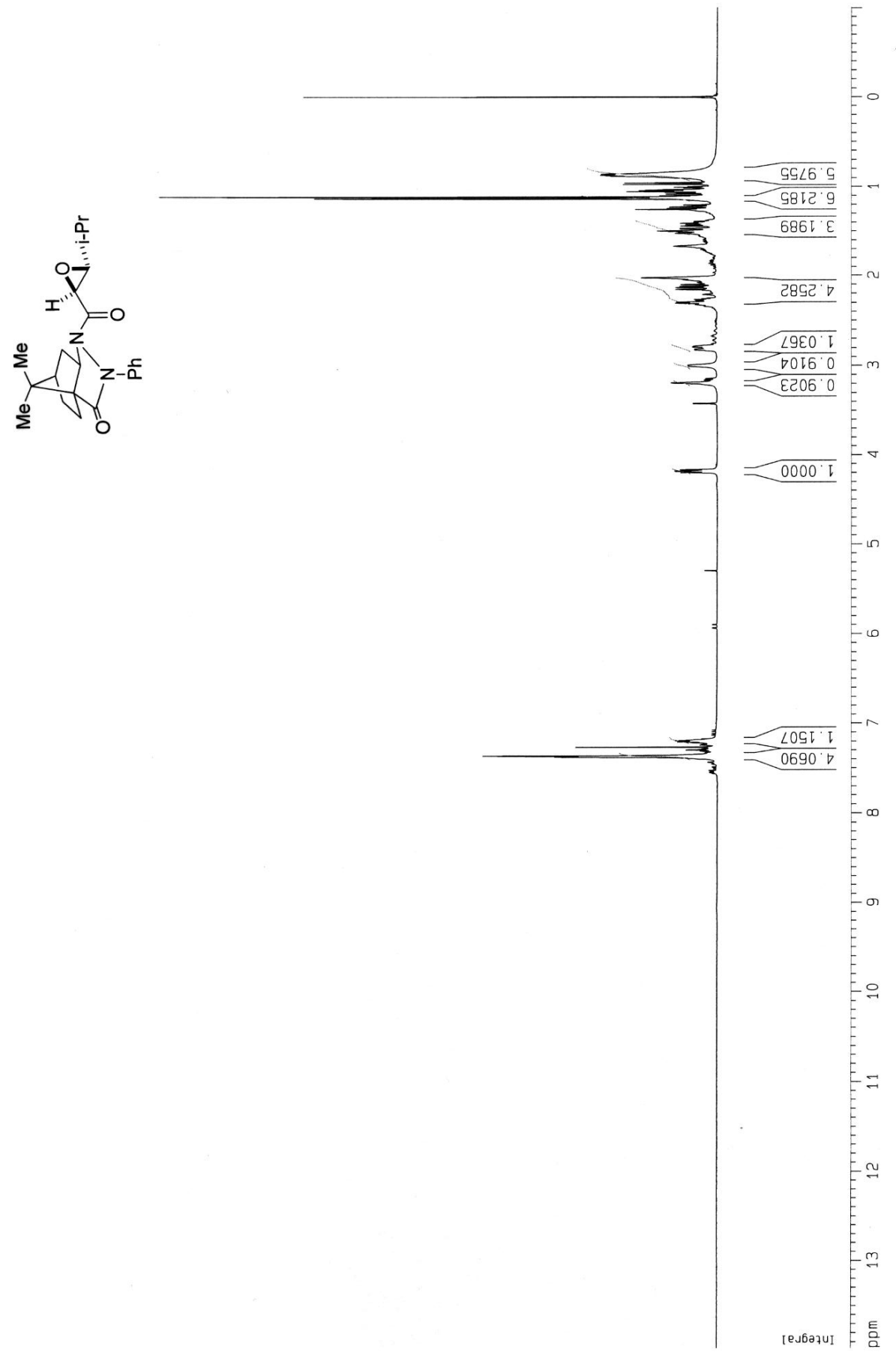




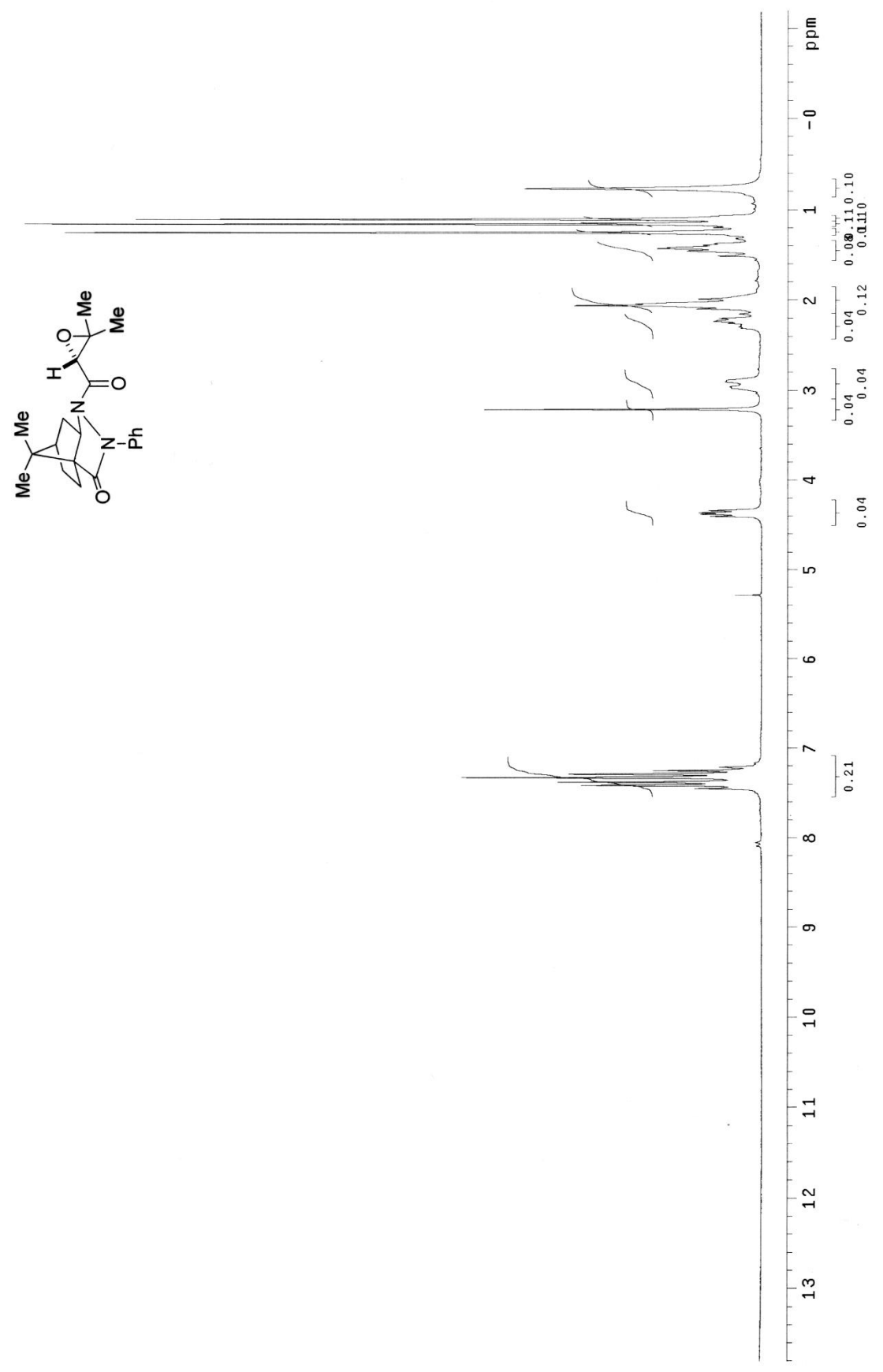



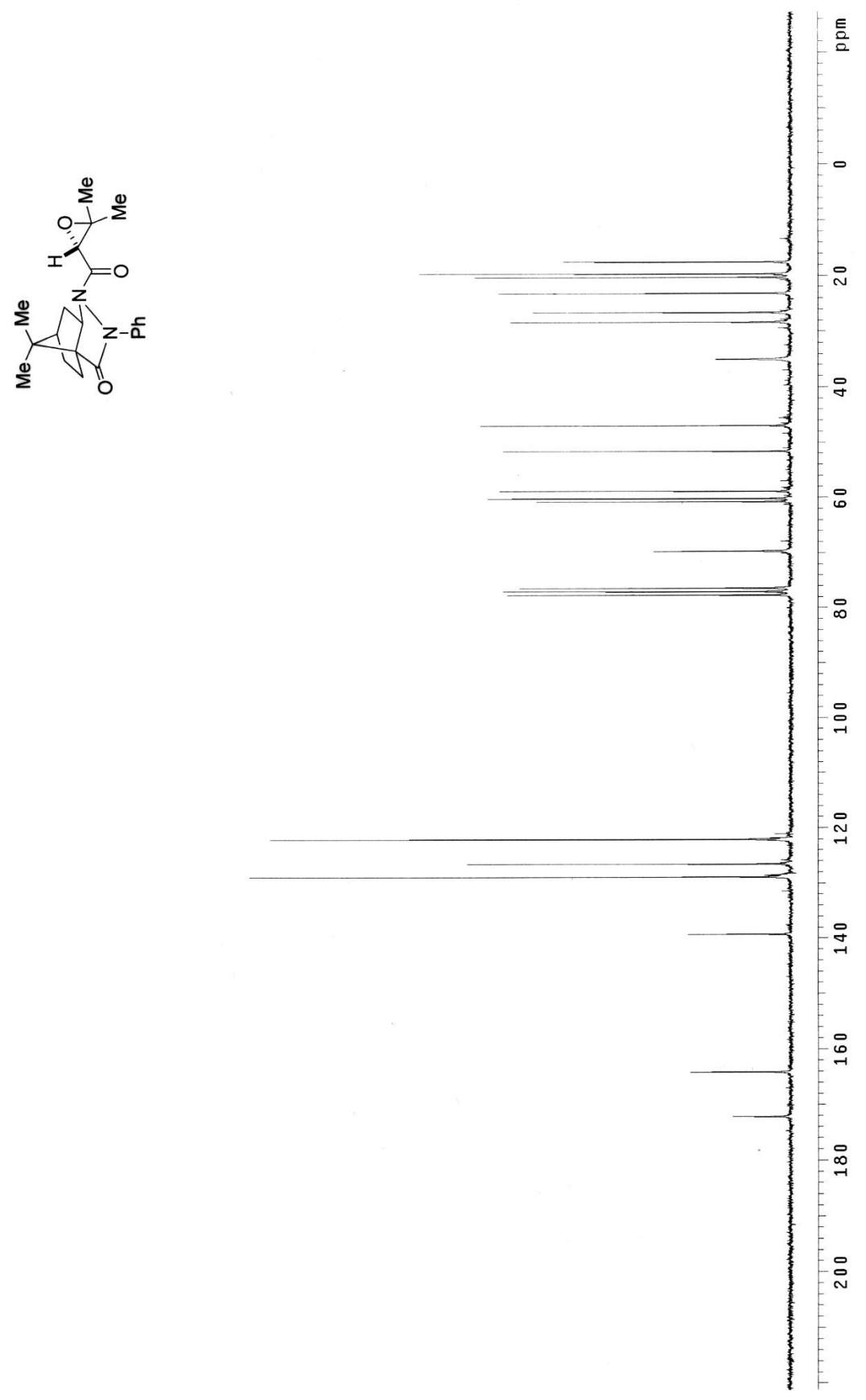


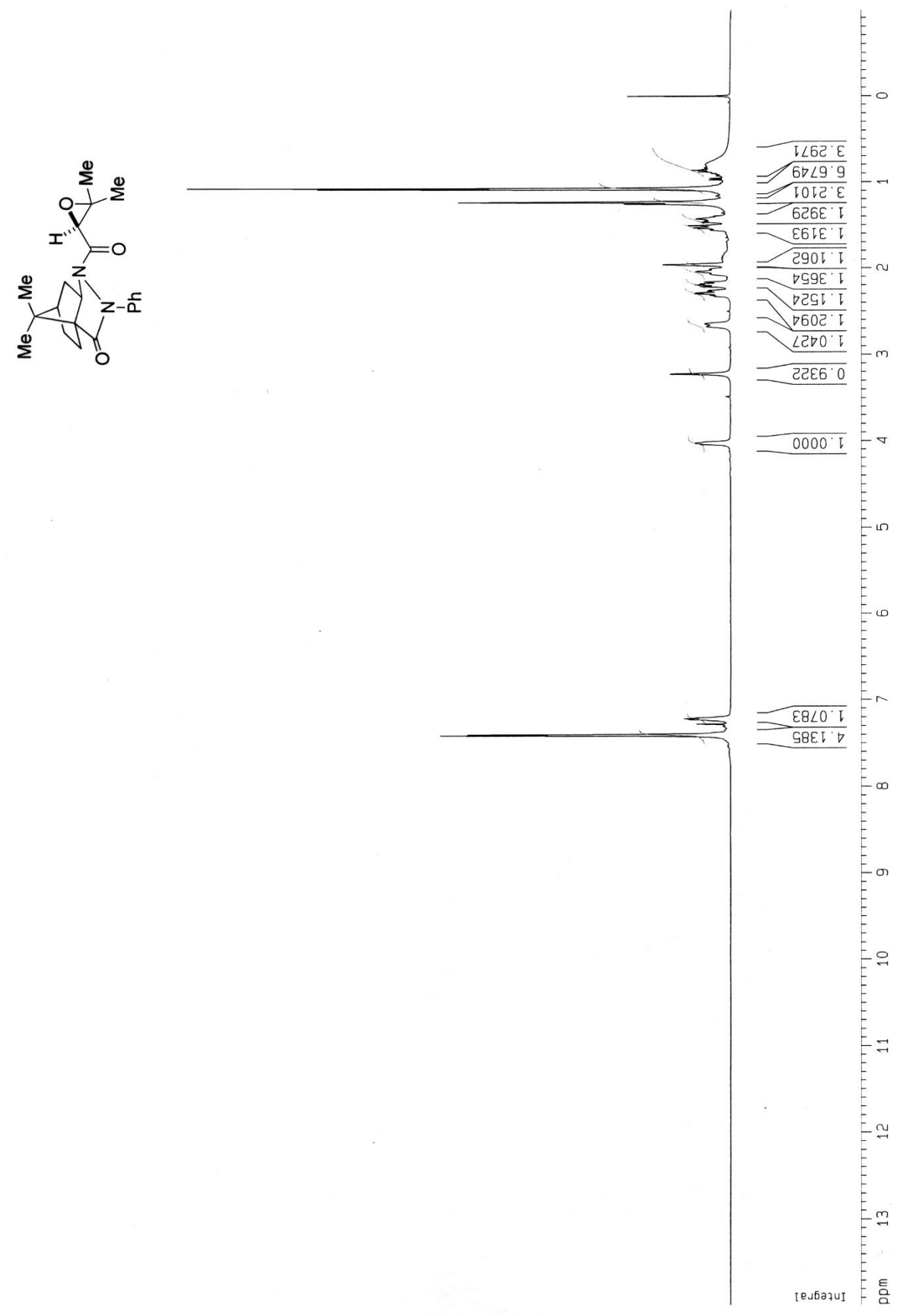




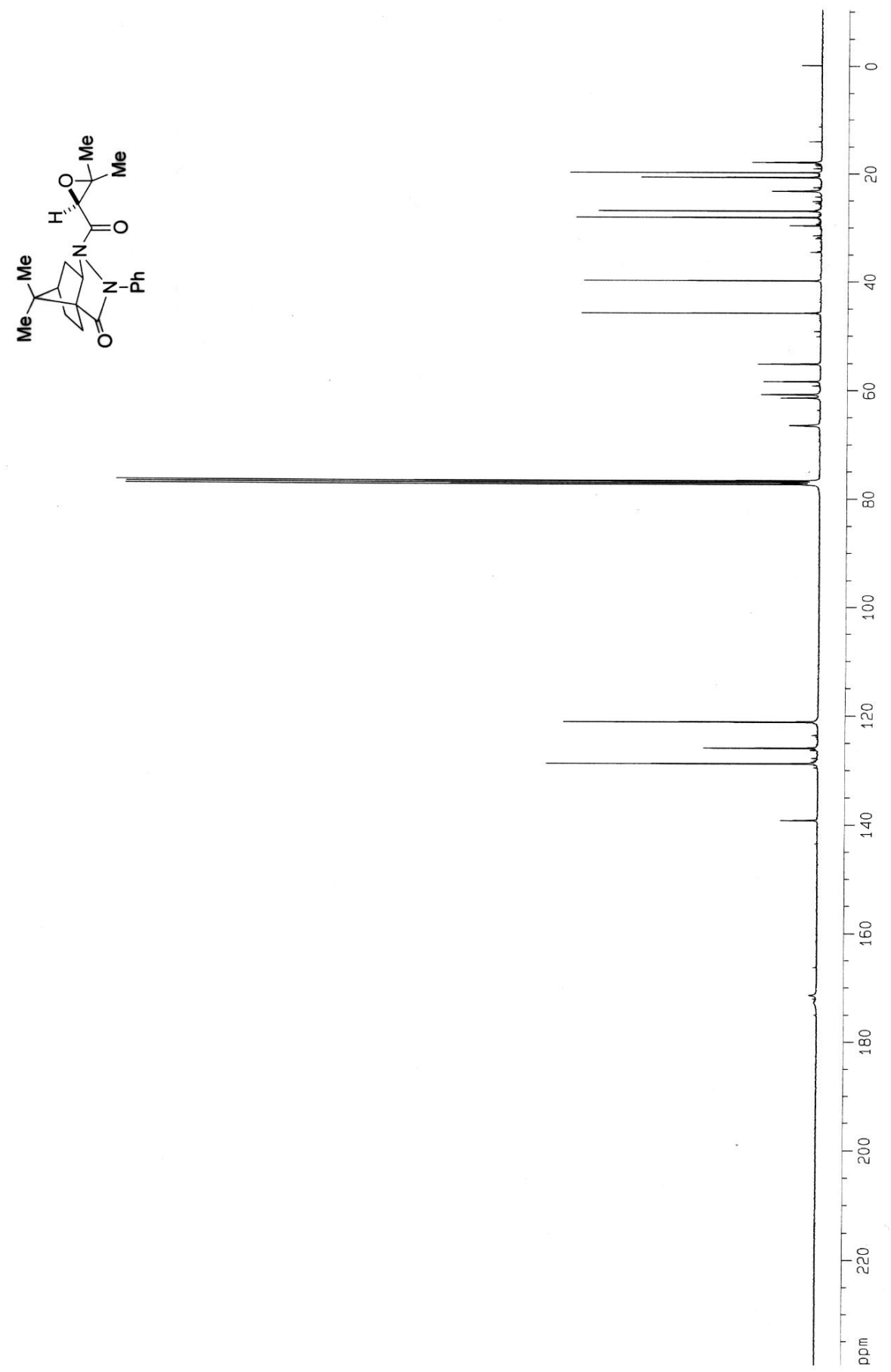




\section{$\operatorname{ch} 2158$}

Abstract

We present the crystal and molecular structure of

\section{Comment}

The study of the titled structure was undertaken to establish its three dimensional structure. Geometries are tabulated below. All diagrams and calculations were performed using maXus (Bruker Nonius, Delft \& MacScience, Japan).

\section{Experimental}

Crystal data

$$
\mathrm{C}_{20} \mathrm{H}_{24} \mathrm{~N}_{2} \mathrm{O}_{3}
$$

$\mathrm{M}_{\mathrm{r}}=340.423$

Monoclinic

$\mathrm{P} 2$ I

$a=10.1762(2) \AA$

$\mathrm{b}=16.5343(4) \AA$

$c=22.0690(7) \AA$

$\alpha=90.00^{\circ}$

$\beta=91.8689(8)^{\circ}$

$y=90.00^{\circ}$

$\mathrm{V}=3711.3(2) \AA^{3}$

$Z=8$

$D_{x}=1.219 \mathrm{Mg} \mathrm{m}^{-3}$

Density measured by: not measured

fine-focus sealed tube

Mo Ka. radiation

$\lambda=0.71073$

Cell parameters from 16501

$\theta=4.076-23.534^{\circ}$

$\mu=0.08 \mathrm{~mm}^{-1}$

$\mathrm{T}=298 \mathrm{~K}$

Cube

Colourless

Crystal source:Local laboratory

Data collection

\section{KappaCCD}

CCD

Absorption correction: multi-scan

$\mathrm{T}_{\min }=0.936, \mathrm{~T}_{\max }=0.994$

23363 measured reflections

5983 independent reflections

4307 observed reflections

Criterion: [> 3.00 sigma(I)

$R_{\text {int }}=0.055$

$\theta_{\max }=23.45^{\circ}$

$h=-11 \rightarrow 11$

$$
\begin{aligned}
& k=-16 \rightarrow 18 \\
& l=-24 \rightarrow 24 \\
& h=0 \rightarrow 11 \\
& k=0 \rightarrow 18 \\
& l=-24 \rightarrow 24
\end{aligned}
$$

Refinement

Refinement on $\mathrm{F}^{2}$

fullmatrix least squares refinement

$R($ all $)=0.085$

$R(g t)=0.056$

$w R$ (ref) $=0.091$

$w R($ all $)=0.100$

$w R(g t)=0.092$

$\mathrm{S}($ ref $)=1.870$

$S($ all $)=1.911$

$\mathrm{S}(\mathrm{gt})=1.906$

4233 reflections

902 parameters

0 restraints

Oniy coordinates of $\mathrm{H}$ atoms refined

Calculated weights sigma

$\Delta / \sigma_{\max }=0.038$

$\Delta \rho_{\max }=0.34 \mathrm{eA}^{3}$

$\Delta \rho_{\min }=-0.37 \mathrm{eA}^{3}$

Extinction correction: Zachariasen

equation(22) p292 'Crystallographic Computing'

(1970) coefficient is multiplied by $10^{4}$

Extinction coefficient $=8(3) \times 105$

Acomic scattering factors from Waasmaier \& Kirfel, 1995

Data collection: KappaCCD

Cell refinement: HKL Scalepack (Otwinowski \& Minor 1997)

Data reduction: Denzo and Scalepak

(Otwinowski \& Minor, 1997)

Program(s) used to refine structure:

maXus(Mackay et al., 1999)

Molecular graphics: ORTEP (Johnson, 1976)

Software used to prepare material for publication:

maXus(Mackay et al., 1999) 

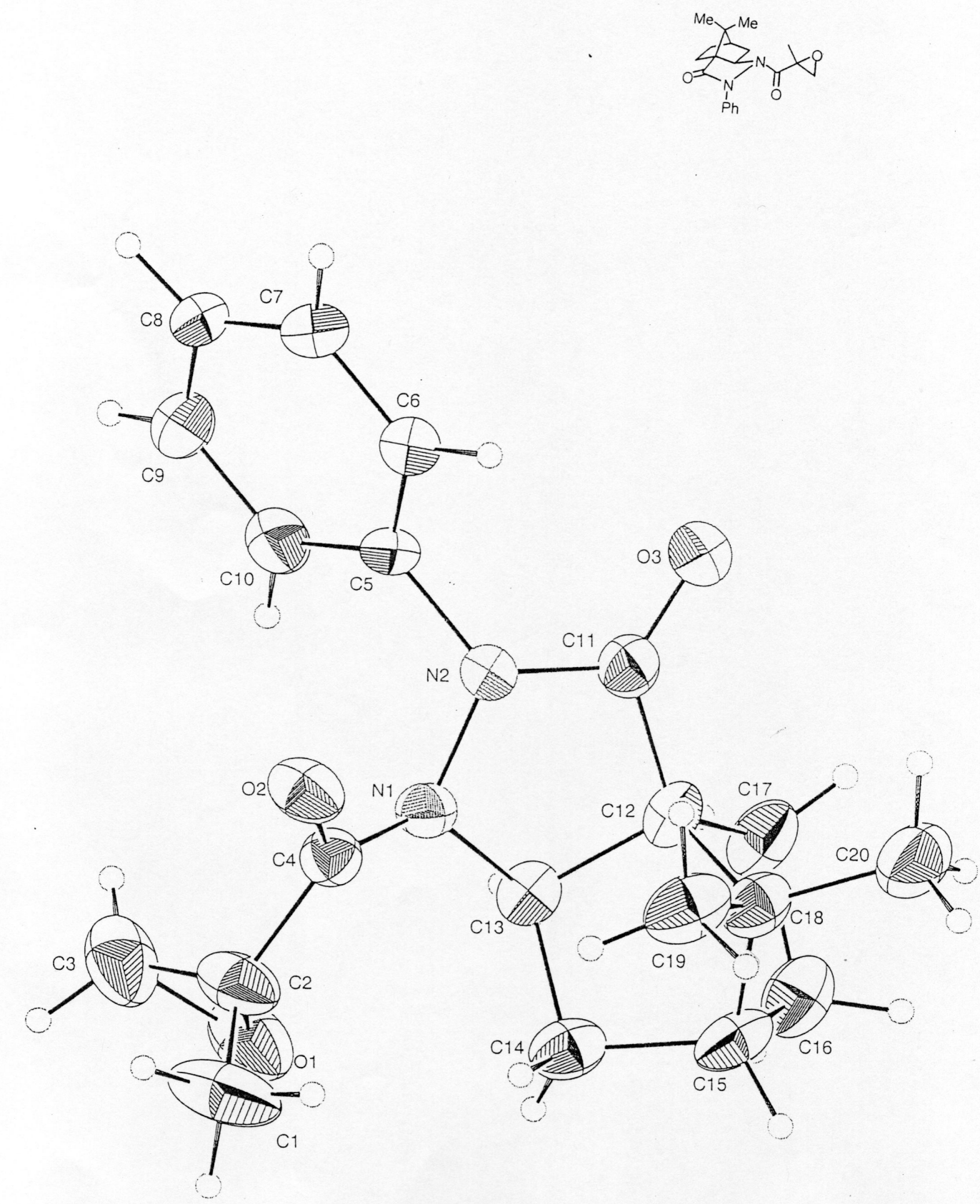


\section{$\operatorname{ch} 2075$}

Abstract
We present the crystal and molecular structure of

Comment

The study of the titled structure was undertaken to establish its three dimensional structure. Geometries are tabulated below. All diagrams and calculations were performed using maXus (Bruker Nonius, Delft \& MacScience, Japan).

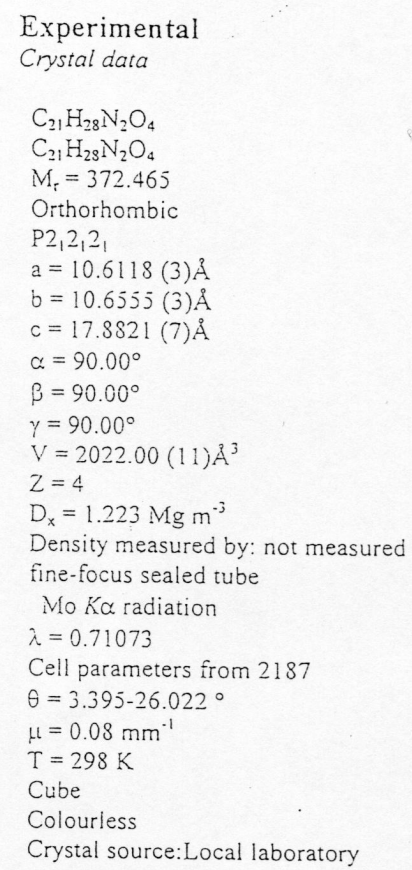

Data collection

KappaCCD

CCD

Absorption correction: none

3890 measured reflections

2340 independent reflections

1700 observed reflections

Criterion: I> 3.00 sigma(I)

$R_{\text {int }}=0.026$

$\theta_{\max }=26.01^{\circ}$

$h=-12 \rightarrow 12$

$k=-13 \rightarrow 13$

$$
\begin{aligned}
& l=-21 \rightarrow 22 \\
& h=0 \rightarrow 12 \\
& k=0 \rightarrow 13 \\
& l=0 \rightarrow 22
\end{aligned}
$$

Refinement

Refinement on $\mathrm{F}^{2}$

fullmatrix least squares refinement

$R($ all $)=0.071$

$R(g t)=0.050$

$w R($ ref $)=0.091$

$w R($ ail $)=0.096$

$w R(g t)=0.091$

$S($ ref $)=1.616$

$S($ ail $)=1.721$

$S(g t)=1.620$

1695 reflections

245 parameters

0 restraints

Only coordinates of $\mathrm{H}$ atoms refined

Calculated weights sigma

$\Delta / \sigma_{\max }=0.019$

$\Delta \rho_{\max }=0.31 \mathrm{eA}^{3}$

$\Delta \rho_{\min }=-0.28 \mathrm{e} \AA^{3}$

Extinction correction: Zachariasen

equation(22) p292 'Crystallographic Computing' $(1970)$ coefficient is multiplied by $10^{4}$
xtinction coefficient $=50(19) \times 104$ Extinction coefficient $=50(19) \times 104$

Atomic scattering factors from Waasmaier \& Kirfel, 1995

Data collection: KappaCCD

Cell refinement: HKL Scalepack (Otwinowski \& Minor 1997)

Data reduction: Denzo and Scalepak

(Otwinowski \& Minor, 1997)

Program(s) used to solve structure: SIR92

(Altomare et al., 1994)

Program(s) used to refine structure:

maXus(Mackay et al., 1999)

Molecular graphics: ORTEP (Johnson, 1976)

Software used to prepare material for publication: 

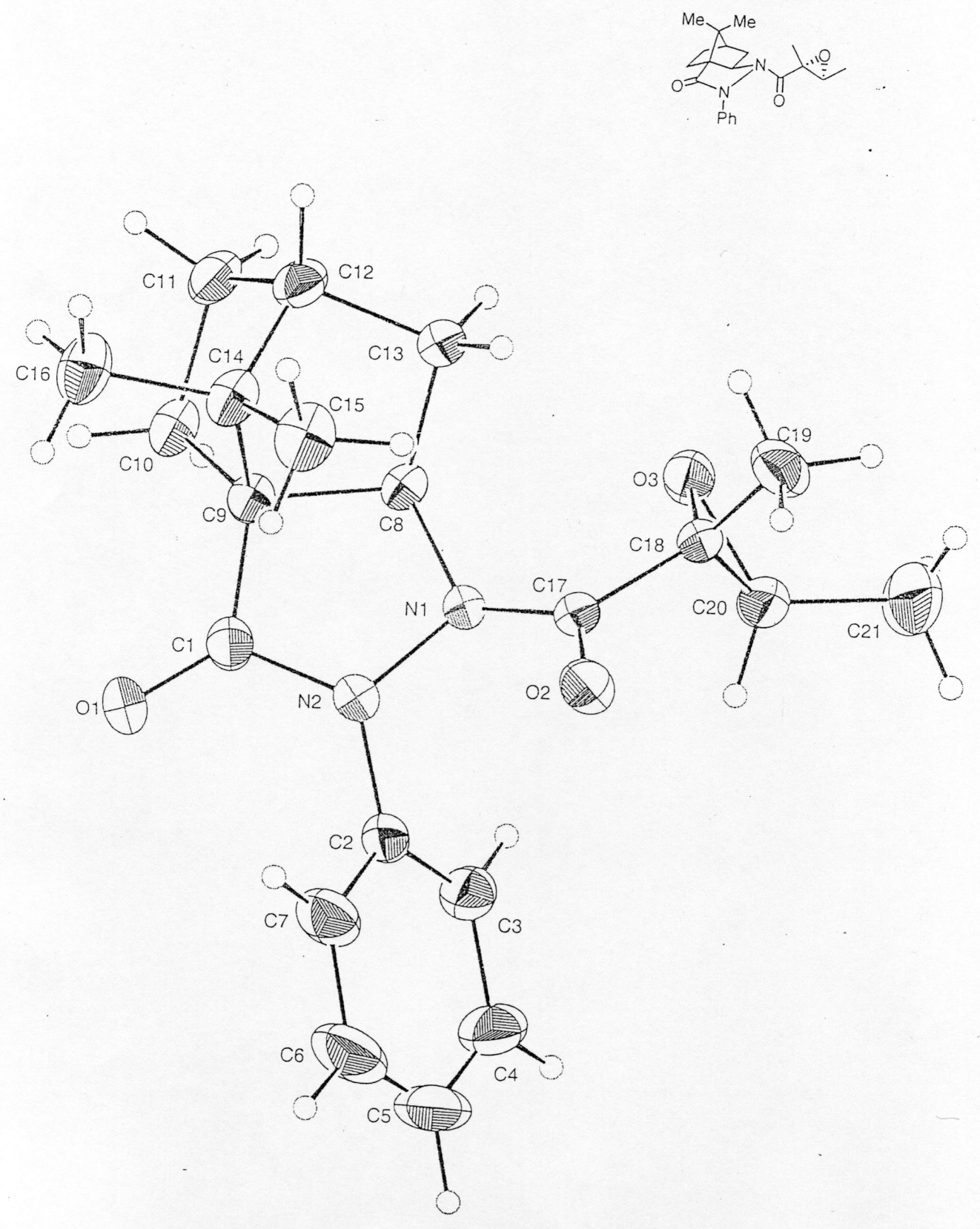
CH2601

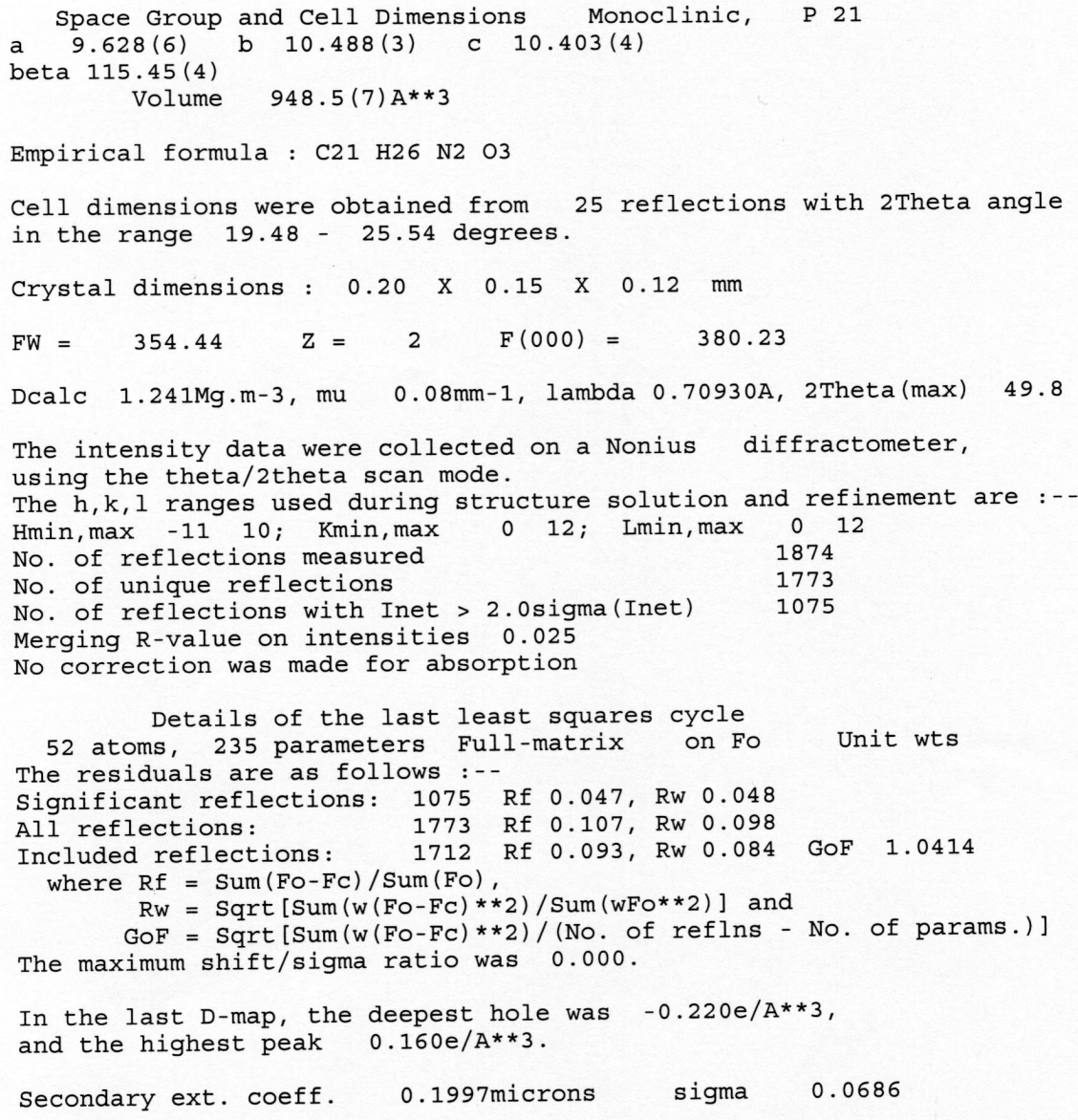



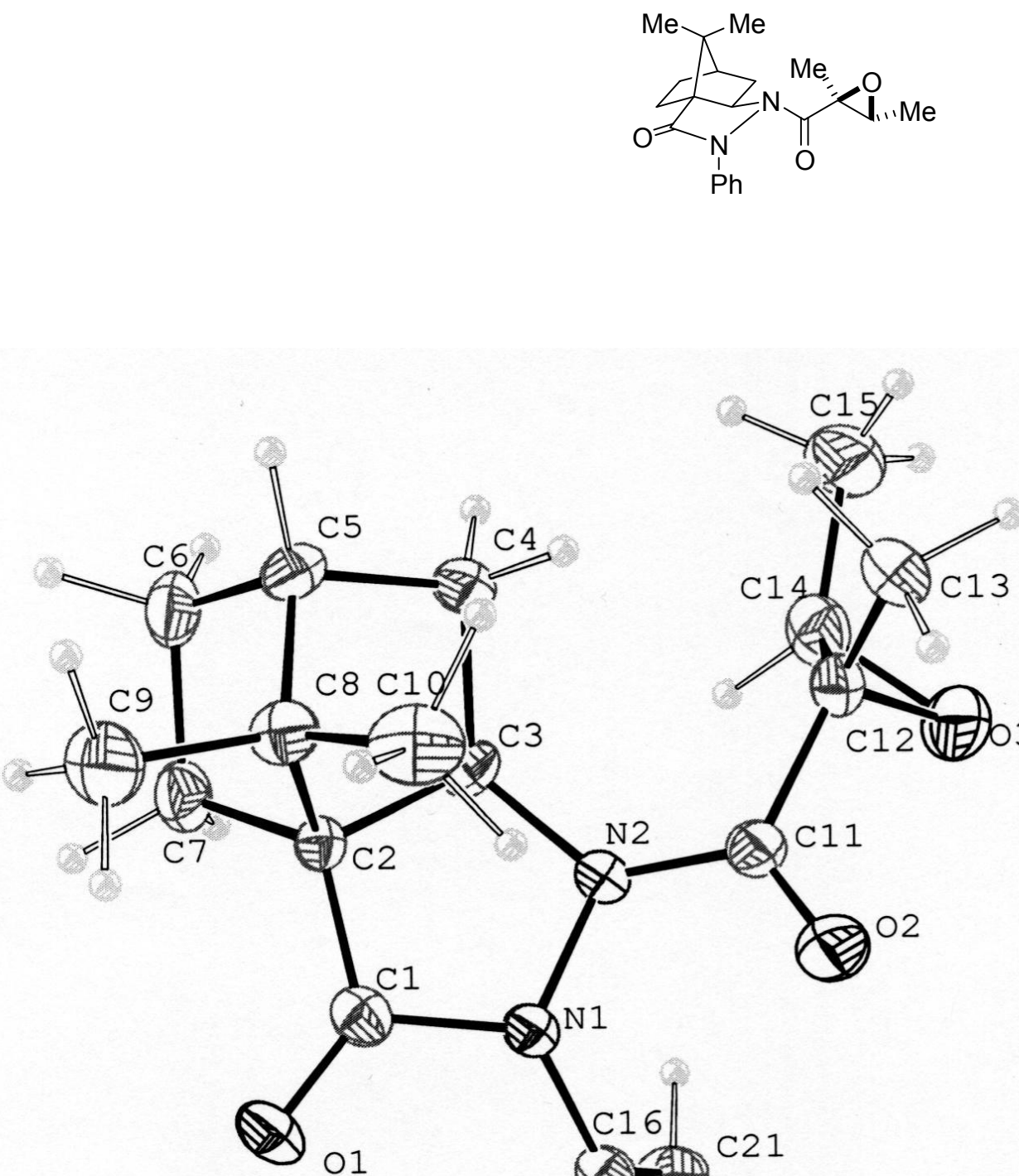

C12(v) 03
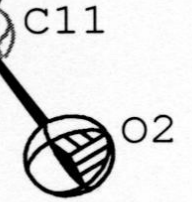

$\mathrm{N} 1$
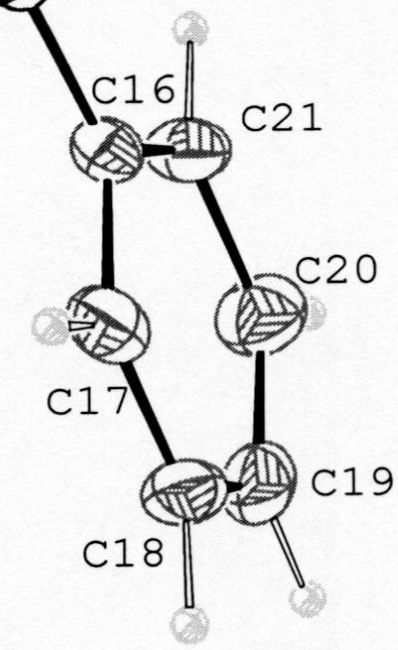


\section{$\operatorname{ch} 2201$}

\begin{tabular}{|c|c|}
\hline \multicolumn{2}{|c|}{ Abstract } \\
\hline \multirow{2}{*}{\multicolumn{2}{|c|}{$\begin{array}{l}\text { Comment } \\
\text { The study of the titled structure was undertaken to establish its three dimensional structure. Geometries are } \\
\text { tabulated below. All diagrams and calculations were performed using maXus (Bruker Nonius, Delft \& } \\
\text { MacScience, Japan). }\end{array}$}} \\
\hline & \\
\hline & $h=-10 \quad 10$ \\
\hline \multirow{3}{*}{$\begin{array}{l}\text { Experimental } \\
\text { Crystal data }\end{array}$} & $k=-10 \quad 11$ \\
\hline & $1=-22 \quad 23$ \\
\hline & $\mathrm{h}=0 \quad 10$ \\
\hline \multirow{3}{*}{\multicolumn{2}{|c|}{$\begin{array}{l}\mathrm{C}_{22} \mathrm{H}_{28} \mathrm{~N}_{2} \mathrm{O}_{3} \\
\mathrm{C}_{22} \mathrm{H}_{28} \mathrm{~N}_{2} \mathrm{O}_{3} \\
\mathrm{M}_{\mathrm{r}}=368.477\end{array}$}} \\
\hline & \\
\hline & \\
\hline \multicolumn{2}{|l|}{$\begin{array}{l}\text { Monoclinic } \\
\mathrm{P} 2_{1}\end{array}$} \\
\hline \multicolumn{2}{|l|}{$a=9.4797(5) \AA$} \\
\hline $\mathrm{b}=10.5020(6) \AA$ & fullmatrix least squares refinement \\
\hline$c=20.749(2) \AA$ & $\mathrm{R}(\mathrm{all})=0.122$ \\
\hline$=90.00^{\circ}$ & $R(g t)=0.060$ \\
\hline$=88.91^{\circ}$ & $w R(r e f)=0.100$ \\
\hline$=90.00^{\circ}$ & $w R($ all $)=0.121$ \\
\hline$V=2065.3(2) \AA^{3}$ & $w R(g t)=0.101$ \\
\hline$Z=4$ & $\mathrm{~S}(\mathrm{ref})=1.906$ \\
\hline $\mathrm{D}_{\mathrm{x}}=1.185 \mathrm{Mg} \mathrm{m}^{-3}$ & $S($ all $)=1.878$ \\
\hline Density measured by: not measured & $\mathrm{S}(\mathrm{gt})=1.937$ \\
\hline fine-focus sealed tube & 1970 reflections \\
\hline Mo $K \quad$ radiation & 488 parameters \\
\hline$=0.71073$ & 0 restraints \\
\hline Cell parameters from 14723 & Only coordinates of $\mathrm{H}$ atoms refined \\
\hline$=4.076-24.407^{\circ}$ & Calculated weights sigma \\
\hline$=0.08 \mathrm{~mm}^{-1}$ & $I_{\max }=0.030$ \\
\hline $\mathrm{T}=298 \mathrm{~K}$ & $\max =0.33 \mathrm{e} \AA^{3}$ \\
\hline Cube & $\min =-0.38 \mathrm{e} \AA^{3}$ \\
\hline Colourless & Extinction correction: Zachariasen \\
\hline Crystal source:Local laboratory & $\begin{array}{l}\text { equation(22) } p 292 \text { 'Crystallographic Computing' } \\
(1970) \text { coefficient is multiplied by } 10^{4} \\
\text { Extinction coefficient }=10 \text { (2) } \times 105\end{array}$ \\
\hline Data collection & $\begin{array}{l}\text { Extinction coefficient }-10(2) \times 105 \\
\text { Atomic scattering factors from Waasmaier \& Kirfel, } 1995\end{array}$ \\
\hline KappaCCD & \\
\hline $\mathrm{CCD}$ & \\
\hline Absorption correction: multi-scan & Data collection: KappaCCD \\
\hline $\mathrm{T}_{\min }=0.955, \mathrm{~T}_{\max }=1.000$ & Cell refinement: HKL Scalepack (Otwinowski \& \\
\hline 11134 measured reflections & Minor 1997) \\
\hline 3642 independent reflections & Data reduction: Denzo and Scalepak \\
\hline 1998 observed reflections & (Otwinowski \& Minor, 1997) \\
\hline Criterion: $\mathrm{I}>3.00$ sigma(I) & Program(s) used to refine structure: \\
\hline $\mathrm{R}_{\text {int }}=0.071$ & maXus(Mackay et al., 1999) \\
\hline $\max =23.89^{\circ}$ & Molecular graphics: ORTEP (Johnson, 1976) \\
\hline
\end{tabular}



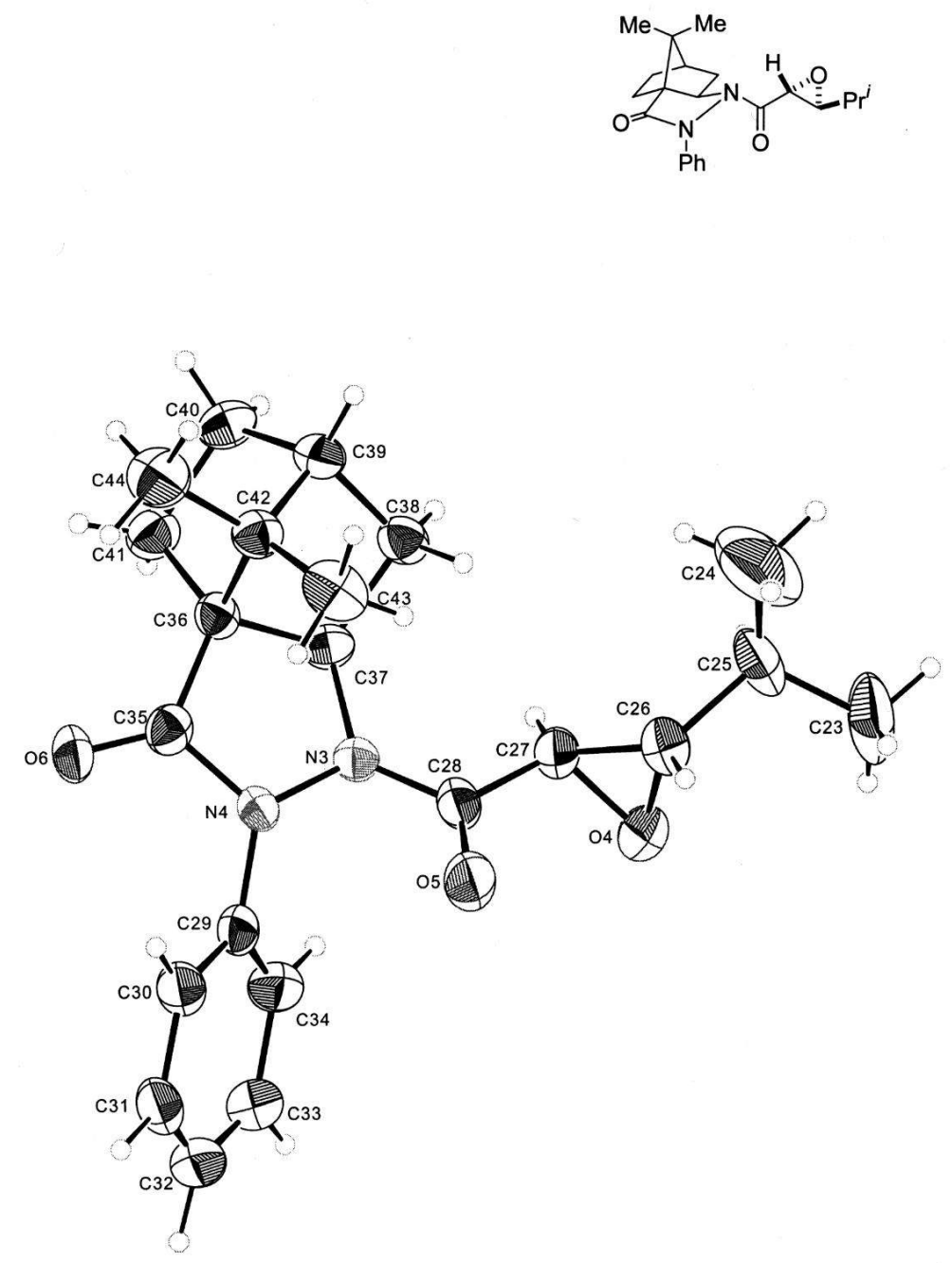
CH1787

Space Group and Cell Dimensions Monoclinic, P 21

a $9.6835(18)$ b $10.287(3)$ c $10.3772(11)$

beta $114.705(13)$

Volume $939.1(3) A^{* * 3}$

Empirical formula : $\mathrm{C} 21 \mathrm{H} 26 \mathrm{~N} 2 \mathrm{O} 3$

Cell dimensions were obtained from 25 reflections with 2Theta angle

in the range $20.94-26.76$ degrees.

Crystal dimensions : $0.60 \times 0.60 \times 0.35 \mathrm{~mm}$

$F W=354.44 \quad Z=2 \quad F(000)=380.23$

Dcalc $1.253 \mathrm{Mg} \cdot \mathrm{m}-3$, mu $0.08 \mathrm{~mm}-1$, lambda $0.70930 \mathrm{~A}$, 2Theta(max) 53.8

The intensity data were collected on a Nonius diffractometer,

using the theta/2theta scan mode.

The h,k,l ranges used during structure solution and refinement are :--

Hmin,max -12 11; Kmin,max 0 13; Lmin,max 013

No. of reflections measured $\quad 2272$

No. of unique reflections 2157

No. of reflections with Inet > 2.0sigma(Inet) 1921

Merging R-value on intensities 0.018

Absorption corrections were made.

The minimum and maximum transmission factors are 0.706170 and 0.978602 .

Details of the last least squares cycle

52 atoms, 235 parameters Full-matrix on Fo Unit wts

The residuals are as follows :-

Significant reflections: 1921 Rf 0.046 , Rw 0.049

All reflections: $\quad 2157$ Rf 0.053 , Rw 0.053

Included reflections: 2118 Rf 0.051 , Rw 0.051 GoF 2.0494

where $\mathrm{Rf}=\operatorname{Sum}(\mathrm{Fo}-\mathrm{Fc}) / \mathrm{Sum}(\mathrm{Fo})$,

$\mathrm{Rw}=\operatorname{Sqrt}\left[\mathrm{Sum}\left(\mathrm{w}\left(\mathrm{F}_{0}-\mathrm{Fc}\right) * * 2\right) / \mathrm{Sum}\left(\mathrm{wF}_{0} * 2\right)\right]$ and

$\mathrm{GoF}=\operatorname{Sqrt}\left[\mathrm{Sum}\left(\mathrm{w}(\mathrm{Fo}-\mathrm{Fc})^{* * 2}\right) /(\right.$ No. of reflns - No. of params. $\left.)\right]$

The maximum shift/sigma ratio was 0.000 .

In the last $\mathrm{D}$-map, the deepest hole was $-0.180 \mathrm{e} / \mathrm{A}^{* * 3}$,

and the highest peak $0.220 \mathrm{e} / \mathrm{A}^{* * 3}$.

Secondary ext. coeff. $\quad 0.9336$ microns sigma $\quad 0.0457$ 

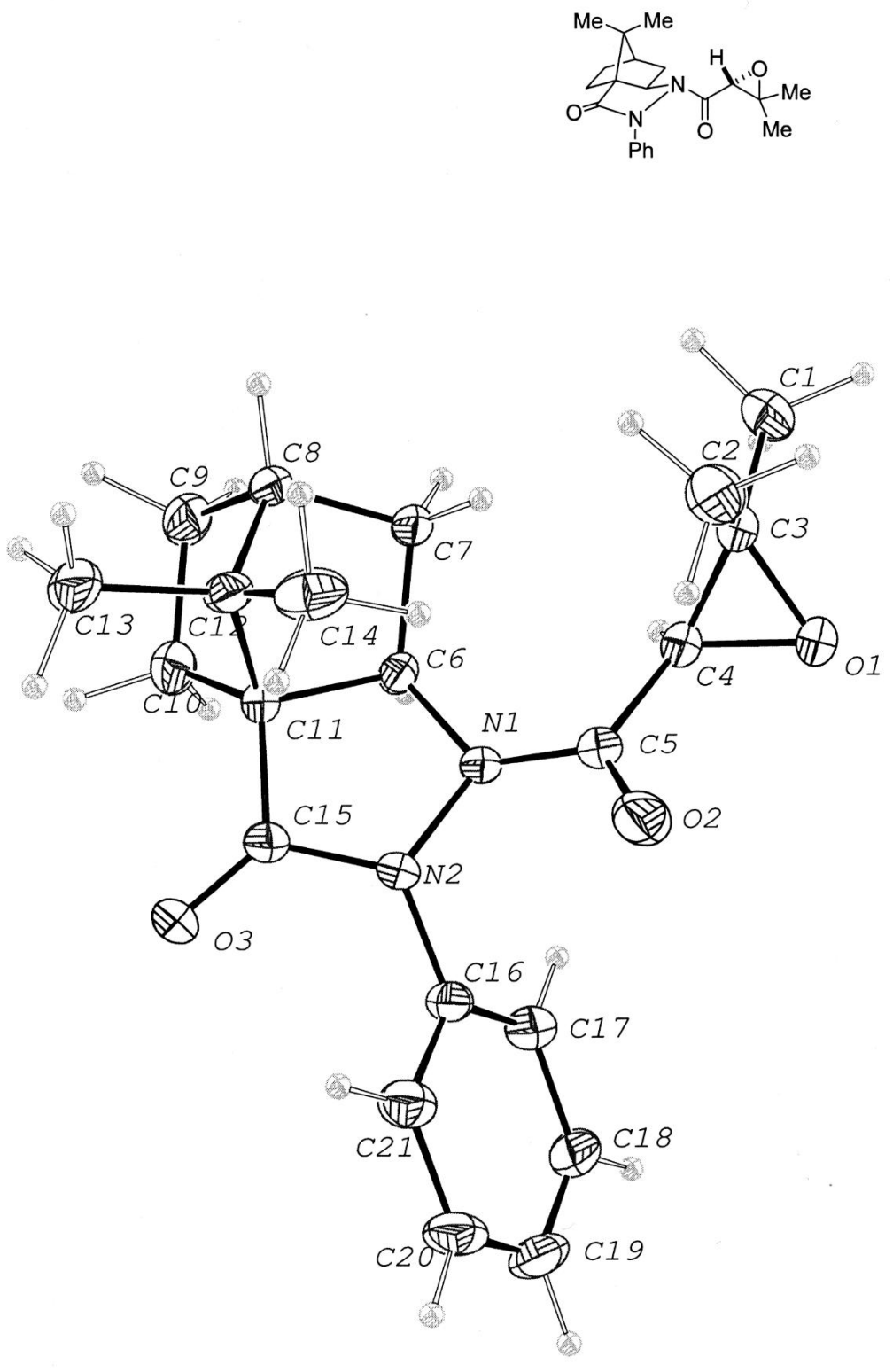